\title{
The Limited Screening Value of Insulin-Like Growth Factor-I as a Marker for Alterations in Body Composition in Very Long-Term Adult Survivors of Childhood Cancer
}

\author{
Karin Blijdorp, $\mathrm{MD}^{1,}{ }^{1,2}$ Marry van den Heuvel-Eibrink, $\mathrm{MD}$, PhD, ${ }^{1}$ Rob Pieters, $\mathrm{MD}$, PhD, ${ }^{1}$ Annemieke Boot, $\mathrm{MD}$, PhD, ${ }^{3}$ \\ Johanna Sluimer, $\mathrm{MLS}^{4}{ }^{4}$ Aart-Jan van der Lelij, MD, $\mathrm{PhD}^{2}$ and Sebastian Neggers, $\mathrm{MD}, \mathrm{PhD}^{1,2}$
}

\begin{abstract}
Background. The clinical relevance of low IGF-I levels, caused by cranial radiotherapy, in adult childhood cancer survivors has not been studied extensively. We evaluated whether IGF-I is a useful marker for altered body composition and growth hormone deficiency (GHD) in this group. Procedure. We analyzed retrospective data from 610 adult childhood cancer survivors, retrieved from the late effects clinic. Median age at diagnosis was 6 years (interquartile range 3-11) and follow-up time was 18 years (13-24). We assessed IGF-I standard deviation scores (SDS), anthropometrical measures, growth hormone stimulation tests in patients with clinical signs of GHD, and measures of body composition (assessed by dual X-ray absorptiometry, Lunar Prodigy). Results. In 58 cranially irradiated acute leukemia survivors (25 Gy (24-25)) and 56 locally irradiated brain tumor survivors (42 Gy (35-54)) we found significantly lower
\end{abstract}

IGF-I SDS $(P<0.001)$, lower height SDS $(P<0.001)$, higher body mass index $(P=0.01)$, higher waist-hip ratio (WHR; $P=0.001)$, higher total fat percentage SDS $(P<0.001)$, and lower lean body mass SDS $(P<0.001)$, as compared to 452 not cranially irradiated survivors. IGF-I showed a weak inverse correlation with BMI $(r=-0.12, P=0.04)$, WHR $(r=-0.15, P=0.01)$, total fat percentage $(r=-0.14, P=0.02)$, and a positive correlation with lean body mass $(r=0.15, P=0.01)$. In patients with low IGF-I levels, IGF-I did not significantly differ between subjects with and without GHD as determined by $\mathrm{GH}$-stimulation testing $(P=0.39)$. Conclusion. This study shows that IGF-I has limited value as a marker for alterations in body composition in adult childhood cancer survivors. Pediatr Blood Cancer 2012;59:711-716.

(c) 2011 Wiley Periodicals, Inc.

Key words: adult childhood cancer survivors; body composition; insulin-like growth factor-I

\section{INTRODUCTION}

Childhood cancer survival rates have improved significantly, with $70-80 \%$ of patients becoming long-term survivors [1]. It has been estimated that 1 out of 640 young adults in the U.S. is a survivor of childhood cancer [2]. Consequently, the incidence of late, treatment-related complications is increasing. Endocrine sequelae, such as the metabolic syndrome, osteopenia, subfertility, thyroid dysfunction, and growth hormone deficiency (GHD), represent an important category of such late effects [3-8].

GHD, which has an incidence of $29-39 \%$ after CRT treatment [9], has many effects on the metabolic system, such as decreased lean body mass and impaired bone mineral density, increased abdominal fat mass, dyslipidemia, insulin resistance, and subsequent raised cardiovascular morbidity later in life [5]. GHD, due to damage of the radiosensitive hypothalamo-pituitary region by CRT, has been associated with low IGF-I levels in adult childhood cancer survivors $[6,10]$. However, it has been shown that IGF-I is a poor marker for GHD in patients treated with CRT [11] and that only very low levels of IGF-I are predictive of GHD [12].

In a previous study in 114 childhood acute lymphoblastic leukemia (ALL) survivors that were treated with CNS irradiation, it was shown that low IGF-I levels were associated with altered body composition, represented by decreased lean body mass, increased total fat percentage, and $65 \%$ more visceral fat in combination with a worsened metabolic risk profile, represented by insulin resistance, and dyslipidemia [13]. This study confirmed results of earlier studies of CRT treated ALL survivors that showed low IGF-I levels associated with total and visceral fat percentage, but not with BMI [6,14]. However, most studies focused on relatively small groups of ALL survivors and after CRT treatment. The value of IGF-I as a marker for body composition, and its association with GHD, is uncertain. Therefore, in this study we analyzed IGF-I levels in a single center cohort of 610 adult childhood cancer survivors.

\section{PATIENTS AND METHODS}

\section{Patients}

We performed a retrospective single center study. Follow-up at our adult late effects outpatient clinic for long-term childhood cancer survivors starts 5 years after cessation of therapy and consultation is individualized, based on cancer diagnosis and treatment protocol. Out of 885 adult childhood cancer survivors, diagnosed and treated between 1964 and 2005, we identified and included 610 survivors, in which IGF-I had been measured between 2004 and 2009, at least 5 years after cessation of cancer treatment. Twenty survivors were living abroad at the time of follow-up, 21 refused to participate and 201 survivors did not show up at the outpatient clinic, due to the fact that the late effects clinic was a new setting and survivors could be under treatment by another specialist. Up until 2009, IGF-I levels were assessed based on former diagnosis and treatment as routine follow-up screening for GHD. Thereafter, according to the national guidelines of screening for late effects as implemented in 2010

\footnotetext{
${ }^{1}$ Department of Pediatric Oncology/Hematology, Erasmus MCSophia Children's Hospital, Rotterdam, The Netherlands; ${ }^{2}$ Department of Medicine, Erasmus MC-University Medical Center Rotterdam, The Netherlands; ${ }^{3}$ Department of Pediatric Endocrinology, Academic Medical Centre Groningen (UMCG), The Netherlands; ${ }^{4}$ Department of Nuclear Medicine, Erasmus MC Rotterdam, The Netherlands

Conflict of interest: Nothing to declare.

*Correspondence to: Karin Blijdorp, MD, Department of Internal Medicine/Endocrinology and Pediatric Oncology/Hematology, Erasmus University Medical Center, P.O. Box 2040, 3000 CA Rotterdam, The Netherlands. E-mail: k.blijdorp@erasmusmc.nl
}

Received 1 September 2011; Accepted 1 November 2011 
by the Dutch Childhood Oncology Group (DCOG), routine measurement of IGF-I levels was omitted.

\section{Clinical Parameters}

Data concerning treatment protocols, as well as disease and patient characteristics, were retrieved from our local database and completed from the medical records where necessary (Table I). Follow-up time was defined as time between cessation of therapy and the most recent visit. Follow-up data of the most recent visit were evaluated including the following variables: use of medication, weight, height, target height $(\mathrm{TH})$ calculated from the height of the survivors' parents [15], body mass index (BMI) calculated from height and weight [16], and waist-hip ratio (WHR) as measured by waist circumference divided by hip circumference [17]. Final height standard deviation score (SDS) was calculated using reference values for Dutch adults: a mean (SD) of 184.0 (7.1) $\mathrm{cm}$ for males and $170.6(6.5) \mathrm{cm}$ for females [15].

\section{Body Composition}

Data on total body fat mass $(\mathrm{kg})$, lean body mass $(\mathrm{kg})$, and percentage of body fat were retrieved from dual energy X-ray absorptiometry (DXA, Lunar Prodigy, GE Healthcare, Madison, WI) (measured in 422 survivors) and assessed as previously described, on the same day as IGF-I measurement. In addition, visceral fat percentage was calculated from intra-abdominal fat $(\mathrm{kg})$ and total fat $(\mathrm{kg})$ using the DXA scan [18]. Values for lean body mass and total fat percentage were compared with normal Dutch reference values and calculated as SDS [19]. WHR and visceral fat percentage were not analyzed in the subset of survivors treated with abdominal radiotherapy because of detrimental local effects on body fat and the frequent occurrence of scoliosis.

\section{Laboratory Assessments}

IGF-I was assessed by Immulite 2000 (DPC Biermann GmbH/ Siemens, Fernwald, Germany), a solid-phase, enzyme-labeled chemiluminescent immunometric assay, with an intra assay variability of $2-5 \%$, and an inter assay variability of 3-7\% [16]. IGF-I levels were compared with reference values by using SDS [20]. In cases where GHD was suspected (i.e., IGF-I below -2 SDS) either insulin tolerance tests or, if contra-indications were present (diabetes mellitus and heart failure), GHRH-Arginine tests were performed [12]. However, only 28 out of the 85 survivors with an IGF-I below -2 SDS were tested for GHD. Eight out of these 28 survivors were tested before 18 years of age because of growth impairment during childhood. One possible reason that only a proportion of the survivors with IGF-I below -2 SDS were tested for GHD is that some endocrinologists are reluctant to treat childhood cancer survivors with growth hormone because of the resultant high recurrence rate [21].

\section{Treatment Groups}

Baseline characteristics of the included survivors are shown in Table I. Three hundred and eighty one survivors had been treated with chemotherapy only. Seventy-three subjects, mainly brain tumor survivors, had been treated with cranial irradiation focused on the tumor field (tumor field CRT). Fifty-seven subjects, mainly leukemia survivors, had been treated with irradiation of the whole cranium (CRT). Twenty survivors had received additional irradiation of the spinal cord. In three leukemia survivors both cranial irradiation and total body irradiation (TBI) were administered. The following treatment groups were analyzed separately after excluding survivors that were treated with growth hormone at the time of the study $(\mathrm{n}=23)$ : CRT treated leukemia survivors $(25$ Gy (24-25)), including 57 CRT survivors, plus three survivors that had been treated with CRT and TBI, minus two subjects that received GH therapy at follow-up $(\mathrm{n}=58)$; survivors treated with local irradiation of the cranium (mainly brain tumors) (42 Gy (35-54)) $(\mathrm{n}=56)$; and TBI survivors (8 Gy $(8-12))(\mathrm{n}=21)$. These three groups were compared separately with all other survivors $(n=452)$, hereafter, referred to as the not cranially irradiated group.

\section{Confounding Factors}

Oral contraceptives can result in lower IGF-I levels, and impaired thyroid function or impaired gonadal function can alter body composition. No subjects were identified with untreated hypothyroidism. Therefore, in our analysis we only adjusted for hypogonadism, represented by oligo- or amenorrhea in women or low testosterone levels in men $(<9 \mathrm{nmol} / \mathrm{L})$ and the use of oral contraceptives in women. As previous therapy with corticosteroids may influence body composition, we also adjusted for corticosteroid treatment.

\section{Statistics}

All data were expressed as median (interquartile range, IQR) unless specified otherwise. Differences between treatment groups were tested using the Mann-Whitney $U$-test for scaled data and the Chi-squared test or the Fisher's exact test for nominal data. Correlations were tested using partial correlations. Regression analysis was used to correct for possible confounding factors. If no SD scores were available, body composition parameters were adjusted for sex and age. $P$-values are all measured in the twoway classification with $P<0.05$ considered statistically significant. Statistical analysis was performed using SPSS 17.0 software (SPSS, Chicago, IL).

\section{RESULTS}

\section{IGF-I Values}

IGF-I SDS of the total group was -0.35 (range $-5.55-2.55$ ), which indicates that IGF-I in this group of adult childhood cancer survivors was lower compared to normal subjects. After correction for possible confounders, both cranially irradiated leukemia survivors and brain tumor irradiated survivors had significantly lower IGF-I SDS, as compared to those treated without cranial irradiation $(P<0.001)$ (Table III). No significant difference in IGF-I SDS was found when comparing the TBI/BMT group with the not cranially irradiated group $(P=0.06)$. Compared with not irradiated survivors, the risk of having an IGF-I SDS below -1 is 4.3 times higher in CRT survivors and 3.2 times higher in survivors treated with localized irradiation therapy for brain tumors (OR $4.3 P<0.001$ and OR 3.2, $P<0.001$ ). 


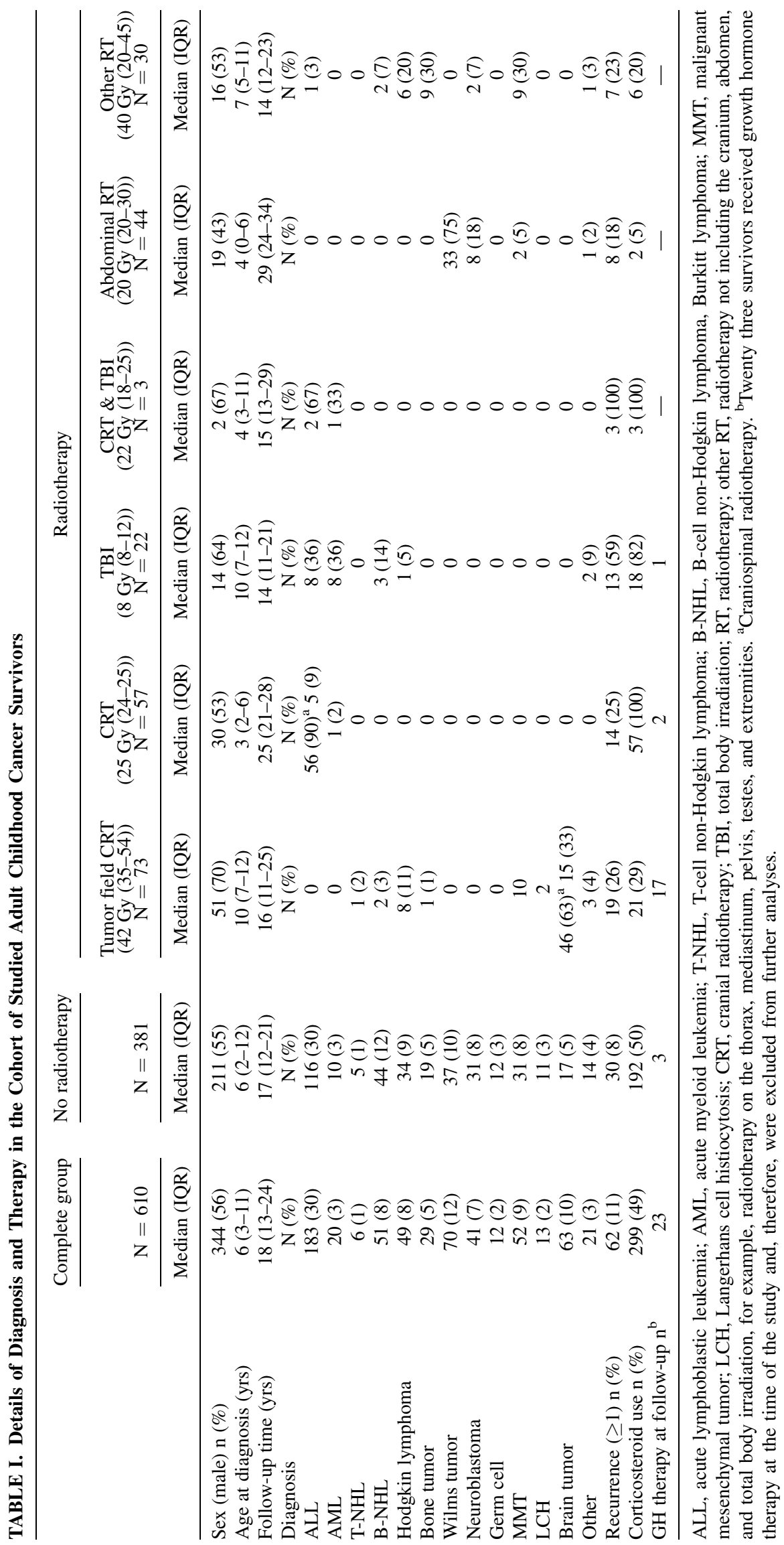




\section{IGF-I and Growth Hormone Deficiency}

Twenty-eight out of the 85 subjects with an IGF-I below -2 SDS were tested with a growth hormone stimulation test, as described earlier (a GHRH-Arginine test was performed in 11 survivors). Seventeen patients were diagnosed with severe GHD by growth hormone stimulation testing, defined as a GH peak $<3 \mu \mathrm{g} / \mathrm{L}$ (insulin tolerance test) or $<9 \mu \mathrm{g} / \mathrm{L}$ (GHRH-Arginine test) [22], whereas, 11 subjects did not have GHD. The 17 GHD subjects had a median IGF-I SDS of $-4.6(-5.7 ;-2.3)$, the 11 non-GHD subjects had a median IGF-I SDS of $-3.8(-5.6$; $-2.7),(P=0.39)$.

\section{Final Height and Body Composition}

Raw data on height and body composition data of the total cohort are depicted in Table II. Adjusted regression coefficients of the different radiotherapy groups as compared to the not cranially irradiated group are depicted in Table III, which is referred to in the following section. Survivors treated with cranial irradiation and irradiated brain tumor patients had significantly lower height SDS $(P<0.001)$ and higher total fat percentage SDS $(P<0.001)$. Leukemia survivors treated with cranial irradiation had higher BMI $(P<0.001)$, higher WHR $(P<0.05)$, and higher visceral fat percentage $(P<0.001)$ than those treated without radiotherapy. Irradiated brain tumor survivors had a higher total fat percentage and lower lean body mass, than those treated without radiotherapy $(P<0.05$ and $P<0.001$, respectively). Survivors treated with TBI had significantly lower height SDS $(P<0.05)$, lower BMI $(P<0.05)$, and lower lean body mass $(P<0.001)$. To correct for $\mathrm{TH}$, the difference between TH SDS and final height SDS was calculated. This was significantly greater in all cranially irradiated groups compared to the not cranially irradiated group $(P<0.001)$.

\section{IGF-I and Body Composition}

After correction for possible confounders, in the whole cohort, IGF-I SDS was positively correlated with height SDS $(\mathrm{r}=0.23$, $P<0.001)$ and lean body mass SDS $(\mathrm{r}=0.15, P=0.01)$; IGF-I SDS was inversely correlated with BMI $(\mathrm{r}=-0.12, P=0.04)$, WHR $(\mathrm{r}=-0.15, P=0.01)$, and total fat percentage SDS ( $\mathrm{r}=-0.14, P=0.02)$. Visceral fat percentage, measured by DXA, was not correlated with IGF-I $(\mathrm{r}=-0.1, P=0.1)$. When analyzing only the subgroup that had been treated with cranial irradiation $(\mathrm{n}=135)$, IGF-I SDS was positively correlated with height $\operatorname{SDS}(\mathrm{r}=0.24, P=0.02)$ and lean body mass (r $=0.23, P=0.03$ ). IGF-I SDS was not significantly correlated with BMI ( $\mathrm{r}=-0.07, P=0.58)$, WHR $(\mathrm{r}=-0.1, P=0.37)$, or total fat percentage $(\mathrm{r}=-0.07, P=0.51)$.

\section{DISCUSSION}

The value of IGF-I as a marker for body composition in longterm childhood cancer survivors has not been studied extensively. Here, we show significantly lower IGF-I SDS and altered body composition in subgroups of survivors, that is, ALL survivors treated with CRT and brain tumor survivors treated with local irradiation. However, IGF-I and all measures of body composition are so weakly correlated that the value of IGF-I as a marker for

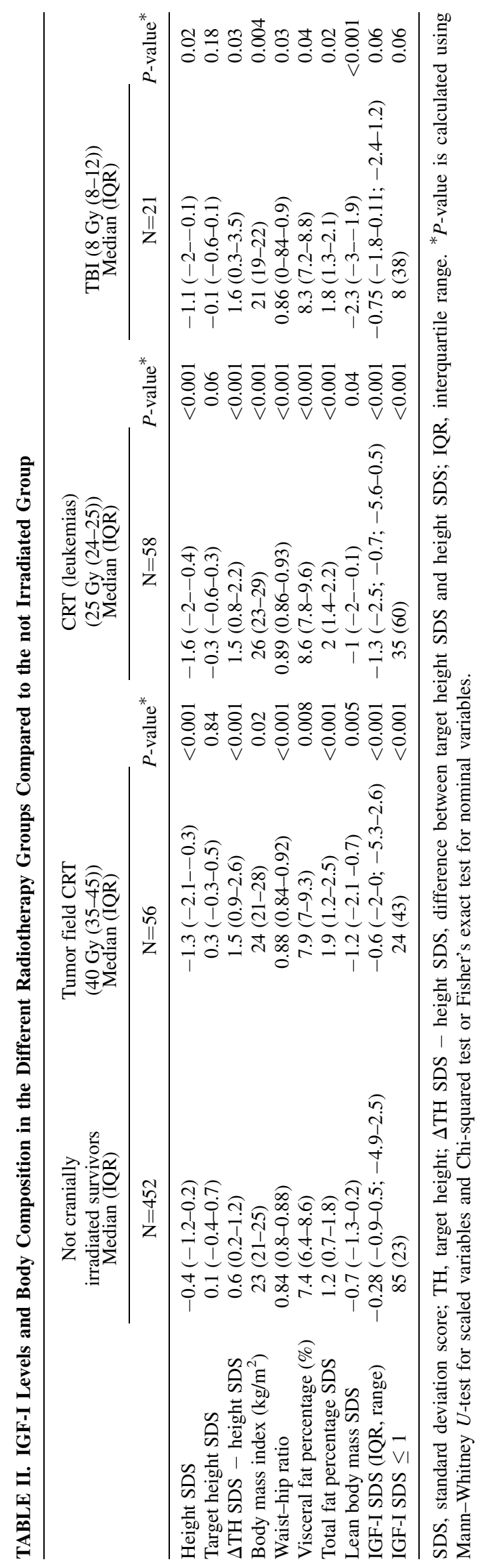


TABLE III. Adjusted Regression Coefficients (95\% CI) of IGF-I Levels and Components of Body Composition of the Different Radiotherapy Groups Compared to the not Cranially Irradiated Group $(n=452)$, Adjusted for Possible Confounders ${ }^{\dagger}$

\begin{tabular}{lcrr}
\hline & Tumor field CRT (42 Gy (35-54)) & CRT (25 Gy (24-25)) & TBI (8 Gy (8-12)) \\
\cline { 2 - 4 } & $\mathrm{N}=56$ & $\mathrm{~N}=58$ & $\mathrm{~N}=21$ \\
\hline Height SDS & $-0.8(-1.2 ;-0.5)^{* *}$ & $-0.7(-1.1 ;-0.4)^{* *}$ & $-0.7(-1.3 ;-0.2)^{*}$ \\
$\Delta$ TH SDS - height SDS & $1.2(0.8-1.7)^{* *}$ & $0.8(0.4-1.2)^{* *}$ & $1.1(0.5-1.8)^{* *}$ \\
Body mass index (kg/m $\left.{ }^{2}\right)$ & $3.7(-12.2-8.9)$ & $11.9(6.8-17.2)^{* *}$ & $-10.2(-16.7 ;-3)^{*}$ \\
Waist-hip ratio & $0.02(0-0.04)$ & $0.04(0.02-0.07)^{*}$ & $0.02(-0.01-0.06)$ \\
Visceral fat percentage (\%) & $-0.2(-0.4-0.8)$ & $1.1(0.6-1.7)^{* *}$ & $0.8(0-1.6)$ \\
Total fat percentage (\%) SDS & $0.4(0.1-0.7)^{*}$ & $0.6(0.3-0.9)^{* *}$ & $0.4(-0.02-0.9)$ \\
Lean body mass (kg) SDS & $-0.7(-1.2 ;-0.3)^{*}$ & $-0.1(-0.5-0.3)$ & $-1.6(-2.2 ;-0.9)^{* *}$ \\
IGF-I SDS & $-0.9(-1.2 ;-0.6)^{* *}$ & $-1.2(-1.5 ;-0.8)^{* *}$ & $-0.2(-0.7-0.4)$ \\
IGF-I SDS $\leq 1$ (OR, 95\% CI) & $3.2(1.7-6)^{* *}$ & $4.3(2.3-8.2)^{* *}$ & $1.4(-0.5-4.1)$ \\
\hline
\end{tabular}

SDS, standard deviation score; TH, target height; $\triangle \mathrm{TH}$ SDS - height SDS difference between target height SDS and height SDS; OR, odds ratio; CI, confidence interval. ${ }^{*} P<0.05 .{ }^{* *} P<0.001$. ${ }^{\dagger}$ Regression coefficient adjusted for sex, age at diagnosis, follow-up time, recurrence, use of oral contraceptives, and hypogonadism (expressed as testosterone $<9 \mathrm{nmol} / \mathrm{L}(\mathrm{m})$ or oligo/amenorrhea without OC use (f)). IGF-I SDS and SD scores are adjusted for all these variables minus sex and follow-up time. Height, body mass index, waist hip ratio, visceral fat percentage, total fat percentage, and lean body mass are also adjusted for treatment with corticosteroids.

clinically relevant alterations in body composition has to be considered insufficient.

In this study we assessed the value of IGF-I as a marker for body composition and GHD. Several studies have shown that IGF-I is the best marker for GH secretory status [23-25]. For this reason, it was initially assumed that IGF-I would also be predictive in the diagnosis of GHD, and therefore IGF-I was used as screening instrument in adult childhood cancer survivors. Our data showed that more than $60 \%$ of the 28 survivors that were tested for GHD turned out to be severely GH deficient, leading us to conclude that low IGF-I is a useful screening instrument. Indeed, pathologically low IGF-I levels (below -2 SDS) have been reported to be a sensitive screening marker, especially in young adults with severe GHD [26]. However, low IGF-I levels do not always represent GHD, because IGF-I levels are dependent upon many other states, such as thyroid function, insulin action, nutritional status, or chronic disease and, therefore, do not fully represent GH status [27]. Furthermore, it is known that IGF-I levels can be artificially normal after CRT treatment because of neurosecretory dysfunction [27] and therefore, the general consensus guideline for adult GHD screening prescribes a mandatory GH stimulation test in cases of clinically suspected GHD [12].

Previous studies in childhood ALL survivors have shown that low IGF-I levels associate with high BMI, high total and visceral fat percentage, and low lean body mass [6,13-14]. BMI is often used to quantify the level of obesity; however, it is known to be only a crude indicator of body fat mass [28-31]. High amounts of total body fat, high visceral fat percentage, and low lean body mass are more accurate markers for true body composition and subsequently are more reliable as risk factors for the development of cardiovascular disease or diabetes mellitus [28-31]. Our study in a large heterogeneous group of adult childhood cancer survivors, confirms that there is a significant negative correlation between IGF-I levels and BMI, WHRs and total fat percentage, and a positive correlation with lean body mass. However, if we focus on the subgroups that had been treated with cranial irradiation, IGF-I SDS showed a stronger positive correlation with lean body mass, whereas, the other measures of body composition were not correlated with IGF-I SDS. However, the correlation coefficients were very weak, meaning that only $2-5 \%$ of the change in body composition is explained by low IGF-I levels.

According to the national guidelines of screening for late effects as implemented by the DCOG, routine measurement of IGF-I levels was omitted, except in groups that are at high risk for GHD, that is, CRT treated ALL survivors and locally irradiated brain tumor survivors [32]. Whereas, low IGF-I levels can guide us to test for GHD, normal IGF-I levels, especially after CRT treatment, do not exclude GHD. Therefore, we recommend that routine IGF-I measurement in these groups be replaced with regular assessment of an insulin tolerance test, which is the gold standard test for GHD. Furthermore, because IGF-I is not a useful marker for clinically relevant alterations in body composition, DEXA scans are recommended in risk groups, as defined in the current study, to assess fat mass and lean body mass.

In this large study we evaluated BMI and body composition in different treatment groups and concluded that after CRT treatment BMI, total fat percentage, WHR, and visceral fat percentage, which is a major risk factor for cardiovascular morbidity, were significantly raised in comparison with not cranially irradiated survivors. Lean body mass was not significantly different. This finding is in agreement with earlier smaller studies in ALL survivors which showed higher BMI, higher total and visceral fat percentage in CRT survivors, in combination with lower lean body mass, which was not found in our CRT survivors [13-14]. This is the first study to measure body composition in a large group of irradiated brain tumor survivors, whereas, most of the previous studies on body composition are in ALL survivors. Alterations in body composition were represented by higher total fat percentage and lower lean body mass, without significantly higher BMI. Gurney et al. [6] showed that brain tumor survivors treated with high dose radiotherapy of the hypothalamus had increased BMI. We did not find raised BMI in irradiated brain tumor survivors, which might be explained by the fact that we did not make a distinction between hypothalamic and non-hypothalamic irradiation. 


\section{CONCLUSION}

This study shows that IGF-I is not of additional value for identifying subjects at risk for alterations in body composition. Patients who had cranial irradiation had higher total fat percentage and lower lean body mass than patients not treated with cranial ìrradiation.

\section{ACKNOWLEDGMENT}

We would like to thank Dr. Patric Delhanty and Mrs. Rina de Wolff for their support in English writing.

\section{REFERENCES}

1. Bozzola M, Locatelli F, Gambarana D, et al. Effect of corticoid therapy on growth hormone secretion. Horm Res 1991;36:183-186

2. Tonorezos ES, Oeffinger KC. Survivorship after childhood, adolescent, and young adult cancer Cancer J 2008;14:388-395.

3. van Waas M, Neggers SJ, Pieters R, et al. Components of the metabolic syndrome in 500 adult longterm survivors of childhood cancer. Ann Oncol 2010;21:1121-1126.

4. van Waas M, Neggers SJ, van der Lelij AJ, et al. The metabolic syndrome in adult survivors of childhood cancer: A review. J Pediatr Hematol Oncol 2010;32:171-179.

5. Steffens M, Beauloye V, Brichard B, et al. Endocrine and metabolic disorders in young adult survivors of childhood acute lymphoblastic leukaemia (ALL) or non-Hodgkin lymphoma (NHL). Clin Endocrinol (Oxf) 2008;69:819-827.

6. Gurney JG, Ness KK, Sibley SD, et al. Metabolic syndrome and growth hormone deficiency in adul survivors of childhood acute lymphoblastic leukemia. Cancer 2006;107:1303-1312.

7. Thomas IH, Donohue JE, Ness KK, et al. Bone mineral density in young adult survivors of acute lymphoblastic leukemia. Cancer 2008:113:3248-3256.

8. Oeffinger KC, Mertens AC, Sklar CA, et al. Chronic health conditions in adult survivors of childhood cancer. N Engl J Med 2006;355:1572-1582.

9. Mulder RL, Kremer LC, van Santen HM, et al. Prevalence and risk factors of radiation-induced growth hormone deficiency in childhood cancer survivors: A systematic review. Cancer Treat Rev 2009;35: 616-632.

10. Adan L, Trivin C, Sainte-Rose C, et al. GH deficiency caused by cranial irradiation during childhood Factors and markers in young adults. J Clin Endocrinol Metab 2001;86:5245-5251.

11. Darzy KH, Shalet SM. Radiation-induced growth hormone deficiency. Horm Res 2003;59:1-11.

12. Growth Hormone Research $\mathrm{S}$. Consensus guidelines for the diagnosis and treatment of growth hormone (GH) deficiency in childhood and adolescence: Summary statement of the GH Research Society. GH Research Society. J Clin Endocrinol Metab 2000;85:3990-3993.
13. Janiszewski PM, Oeffinger KC, Church TS, et al. Abdominal obesity, liver fat, and muscle composition in survivors of childhood acute lymphoblastic leukemia. J Clin Endocrinol Metab 2007:92:3816-3821.

14. Jarfelt M, Lannering B, Bosaeus I, et al. Body composition in young adult survivors of childhood acute lymphoblastic leukaemia. Eur J Endocrinol 2005;153:81-89.

15. Fredriks AM, van Buuren S, Burgmeijer RJ, et al. Continuing positive secular growth change in The Netherlands 1955-1997. Pediatr Res 2000;47:316-323.

16. Health BNIo., National Institutes of Health (NIH), National Heart, Lung, and Blood Institute (NHLBI) The practical guide: Identification, evaluation, and treatment of overweight and obesity in adults. NIH publication 00-40842000.

17. Dobbelsteyn CJ, Joffres MR, MacLean DR, et al. A comparative evaluation of waist circumference, waist-to-hip ratio and body mass index as indicators of cardiovascular risk factors. The Canadian Heart Health Surveys. Int J Obes Relat Metab Disord 2001;25:652-661.

18. Pimenta N, Santa-Clara H, Fragoso IJ. Comparison of body composition and body fat distribution of patients following a cardiac rehabilitation program and sedentary patients. Rev Port Cardiol 2010;29. $1163-1180$

19. van der Sluis IM, de Ridder MA, Boot AM, et al. Reference data for bone density and body composition measured with dual energy $\mathrm{X}$ ray absorptiometry in white children and young adults. Arch Dis Child 2002;87:341-347. discussion 341-347.

20. Elmlinger MW, Kuhnel W, Weber MM, et al. Reference ranges for two automated chemiluminescent assays for serum insulin-like growth factor I (IGF-I) and IGF-binding protein 3 (IGFBP-3). Clin Chem Lab Med 2004;42:654-664.

21. Ergun-Longmire B, Mertens AC, Mitby P, et al. Growth hormone treatment and risk of second neoplasms in the childhood cancer survivor. J Clin Endocrinol Metab 2006;91:3494-3498.

22. Ghigo E, Aimaretti G, Corneli G. Diagnosis of adult GH deficiency. Growth Horm IGF Res 2008:18: $1-16$

23. Blum WF, Albertsson-Wikland K, Rosberg S, et al. Serum levels of insulin-like growth factor I (IGF-I) and IGF binding protein 3 reflect spontaneous growth hormone secretion. J Clin Endocrinol Metab 1993;76:1610-1616.

24. de Boer H, Blok GJ, Van der Veen EA. Clinical aspects of growth hormone deficiency in adults. Endocr Rev 1995;16:63-86.

25. Clemmons DR. Value of insulin-like growth factor system markers in the assessment of growth hormone status. Endocrinol Metab Clin North Am 2007:36:109-129.

26. Shalet SM. Partial growth hormone deficiency in adults: Should we be looking for it? Clin Endocrinol (Oxf) 2010;73:432-435.

27. Murray RD, Bidlingmaier M, Strasburger CJ, et al. The diagnosis of partial growth hormone deficiency in adults with a putative insult to the hypothalamo-pituitary axis. J Clin Endocrinol Metab 2007;92: 1705-1709.

28. Bjorntorp P. Body fat distribution, insulin resistance, and metabolic diseases. Nutrition 1997;13: $795-803$.

29. Larsson B, Svardsudd K, Welin L, et al. Abdominal adipose tissue distribution, obesity, and risk of cardiovascular disease and death: 13 year follow up of participants in the study of men born in 1913. Br Med J (Clin Res Ed) 1984;288:1401-1404.

30. Paradisi G, Smith L, Burtner C, et al. Dual energy X-ray absorptiometry assessment of fat mass distribution and its association with the insulin resistance syndrome. Diabetes Care 1999;22: $1310-1317$

31. Sardinha LB, Teixeira PJ, Guedes DP, et al. Subcutaneous central fat is associated with cardiovascular risk factors in men independently of total fatness and fitness. Metabolism 2000;49:1379-1385.

32. Kremer MWMJ LCM, van Leeuwen FE, Versluys AB, et al. Landelijke richtlijnen voor follow-up van overlevenden van kinderkanker. Tijdschrift voor Kindergeneeskunde 2006;74:247-250. 